\title{
A Remark on the Tree Property in a Choiceless Context ${ }^{* \dagger}$
}

\author{
Arthur W. Apter ${ }^{\ddagger}$ \\ Department of Mathematics \\ Baruch College of CUNY \\ New York, New York 10010 USA \\ and \\ The CUNY Graduate Center, Mathematics \\ 365 Fifth Avenue \\ New York, New York 10016 USA \\ http://faculty.baruch.cuny.edu/apter \\ awapter@alum.mit.edu
}

November 29, 2009

(revised March 7, 2011)

\begin{abstract}
We show that the consistency of the theory "ZF + DC + Every successor cardinal is regular + Every limit cardinal is singular + Every successor cardinal satisfies the tree property" follows from the consistency of a proper class of supercompact cardinals. This extends earlier results due to the author showing that the consistency of the theory " $\mathrm{ZF}+\neg \mathrm{AC}_{\omega}+$ Every successor cardinal is regular + Every limit cardinal is singular + Every successor cardinal satisfies the tree property" follows from hypotheses stronger in consistency strength than a supercompact limit of supercompact cardinals. A lower bound in consistency strength is provided by a result of Busche and Schindler, who showed that the consistency of the theory "ZF + Every successor cardinal is regular + Every limit cardinal is singular + Every successor cardinal satisfies the tree property" implies the consistency of $\mathrm{AD}^{L(R)}$.
\end{abstract}

We begin by very briefly mentioning some of our conventions and terminology. As usual, for $\kappa$ a regular cardinal, a $\kappa$-tree is a tree of height $\kappa$, all of whose levels have cardinality less than $\kappa . \kappa$ satisfies the tree property if every $\kappa$-tree has a branch of length $\kappa$. A $\kappa$-tree not satisfying the tree

*2000 Mathematics Subject Classifications: 03E25, 03E35, 03E45, 03E55.

${ }^{\dagger}$ Keywords: Supercompact cardinal, tree property, indestructibility, symmetric inner model.

¥The author's research was partially supported by PSC-CUNY grants.

$\S$ The author wishes to thank Ralf Schindler for helpful correspondence on the subject matter of this paper which considerably improved and clarified its presentation. 
property is called a $\kappa$-Aronszajn tree. For our purposes, all $\kappa$-trees will be of cardinality $\kappa$ and will have base set $\kappa \times \kappa$. This means that every $\kappa$-tree may be coded by a set of ordinals.

The study of the tree property at successor cardinals is one which has had a long and rich history in set theory. Classically, Aronszajn (see [10, Theorem 9.16, pages 116-117]) showed that assuming the Axiom of Choice, the tree property must fail at $\aleph_{1}$, i.e., that $\aleph_{1}$ carries an $\aleph_{1}$-Aronszajn tree. In his doctoral dissertation [16], Silver demonstrated that if the tree property holds at a cardinal $\kappa>\aleph_{1}$, then $\kappa$ must be weakly compact in $L$. In his doctoral dissertation [14], Mitchell proved that the tree property at the successor of a regular cardinal greater than $\aleph_{1}$ can be forced from a weakly compact cardinal. Taken together, Silver's and Mitchell's results consequently show that the tree property at the successor of a regular cardinal greater than $\aleph_{1}$ is equiconsistent with the existence of a weakly compact cardinal. Abraham [1] demonstrated that relative to the existence of a supercompact cardinal with a weakly compact cardinal above it, it is consistent for $2^{\aleph_{0}}=\aleph_{2}$ and for $\aleph_{2}$ and $\aleph_{3}$ both to satisfy the tree property. Shelah [13] showed that the successor of a singular limit of strongly compact cardinals satisfies the tree property, and Magidor and Shelah together [13] proved that further, relative to a huge cardinal with $\omega$ many supercompact cardinals above it, it is consistent for SCH to hold at $\aleph_{\omega}$ and for $\aleph_{\omega+1}$ to satisfy the tree property. Sinapova [17] improved Magidor and Shelah's result of [13] by constructing a model in which SCH holds at $\aleph_{\omega}$ and $\aleph_{\omega+1}$ satisfies the tree property using only $\omega$ many supercompact cardinals. Cummings and Foreman [6] demonstrated that relative to the existence of $\omega$ many supercompact cardinals, it is consistent for $2^{\aleph_{n}}=\aleph_{n+2}$ for every $n<\omega$ and for every $\aleph_{n}$ for $1<n<\omega$ to satisfy the tree property. Schindler [15] showed that if both $\aleph_{2}$ and $\aleph_{3}$ satisfy the tree property, then there is an inner model with a strong cardinal. Foreman, Magidor, and Schindler [7] proved that if $\aleph_{n}$ has the tree property for all $1<n<\omega$ and $\aleph_{\omega}$ is a strong limit cardinal, then for all $X \in H_{\aleph_{\omega}}$ and all $n<\omega, M_{n}^{\sharp}(X)$ exists. The work of [15] and [7] therefore yields the necessity of strong hypotheses for the aforementioned results of Abraham and Cummings-Foreman. Left open by all of these theorems, however, is the following

Question: Is it possible to combine and extend these results by obtaining a model of ZFC in which 
every successor cardinal greater than $\aleph_{1}$ satisfies the tree property?

Unfortunately, we are unable to answer the above Question in a ZFC context. The purpose of this note is to extend an earlier result (which follows from the main theorem of [4]) and construct a model of ZF + DC in which every successor cardinal satisfies the tree property. Specifically, we prove the following theorem.

Theorem 1 Suppose $V \vDash " Z F C+$ There is a proper class of supercompact cardinals". There is then a partial ordering $\mathbb{P} \subseteq V$ and a symmetric inner model $N \subseteq V^{\mathbb{P}}$ such that $N \vDash " Z F+D C+$ Every successor cardinal is regular + Every limit cardinal is singular + Every successor cardinal satisfies the tree property".

We take this opportunity to make several remarks concerning Theorem 1. A corollary of the main theorem of [4] is the construction, using an almost huge cardinal, ${ }^{1}$ of a model $N^{*}$ for the theory " $\mathrm{ZF}+\neg \mathrm{AC}_{\omega}+$ Every limit cardinal is singular + Every successor cardinal is weakly compact". ${ }^{2}$ The work of [2] shows that the assumption of the existence of an almost huge cardinal may be weakened to a technical hypothesis which is, in consistency strength, strictly in between a supercompact limit of supercompact cardinals and an almost huge cardinal. Since the proof that a weakly compact cardinal satisfies the tree property requires no use of the Axiom of Choice (see, e.g., the proof of $[10$, Lemma $9.26(\mathrm{i})$, page 120$]), N^{*}$ is automatically a model for the theory "ZF $+\neg \mathrm{AC}_{\omega}+$ Every limit cardinal is singular + Every successor cardinal satisfies the tree property". Thus, the model $N$ of Theorem 1 generalizes the model $N^{*}$ of [4] and [2], in the sense that $N$ satisfies a weak fragment of the Axiom of Choice and is constructed using a weaker hypothesis than that found in either [4] or [2]. In addition, in the model $N$ of Theorem 1 , if $\kappa$ is the successor of a limit cardinal, then $\kappa$ is not weakly compact. This is since our construction will guarantee that $V \subseteq N$, so by the way in which $N$ has been built, there is a $\kappa^{+}$sequence of subsets of $\kappa$. By the proofs of [10, Lemmas 9.4 and 9.5, page 110], which require no use of the Axiom of Choice, if there

\footnotetext{
${ }^{1}$ The statement of the main theorem of [4] indicates the use of a 3 huge cardinal. As was pointed out by Moti Gitik, and as was mentioned at the end of [4], an almost huge cardinal suffices for the proof.

${ }^{2}$ In the context of the failure of the Axiom of Choice, the cardinal $\kappa$ is weakly compact if it satisfies the partition relation $\kappa \rightarrow(\kappa)^{2}$.
} 
is a $\kappa^{+}$sequence of subsets of $\kappa$, then $\kappa$ is not weakly compact. This provides another contrast with the model $N^{*}$ of [4] and [2], in which every successor cardinal is weakly compact. Further, by the work of Busche and Schindler [5], $\mathrm{AD}^{L(R)}$ provides a lower bound in consistency strength for the theory "ZF + Every successor cardinal is regular + Every limit cardinal is singular + Every successor cardinal satisfies the tree property". Finally, Theorem 1 illustrates how the situation may differ when the Axiom of Choice is false. In particular, the tree property for $\aleph_{1}$ will hold in the model $N$ witnessing the conclusions of Theorem 1 . As we have already observed, this is impossible when the full Axiom of Choice is true.

Turning now to the proof of Theorem 1, let $V \vDash$ "ZFC + There is a proper class of supercompact cardinals". As in the proof of [3, Theorem 1], we assume without loss of generality that in $V$, each supercompact cardinal $\kappa$ is Laver indestructible [11] under $\kappa$-directed closed forcing, and that there is no inaccessible limit of supercompact cardinals in $V .^{3}$

Our model $N$ witnessing the conclusions of Theorem 1 is the model $N$ of [3, Theorem 1]. In order to describe $N$ more precisely, let $\mathcal{K}=\{\omega\} \cup\{\kappa \mid \kappa$ is either a supercompact cardinal or the successor of a limit of supercompact cardinals $\}$. Assume that $\left\langle\kappa_{i}\right| i \in$ Ord $\rangle$ enumerates $\mathcal{K}$ in increasing order. For each $i \in \operatorname{Ord}$, let $\mathbb{P}_{i}=\operatorname{Coll}\left(\kappa_{i},<\kappa_{i+1}\right)$, i.e., $\mathbb{P}_{i}$ is the Lévy collapse of all cardinals in the open interval $\left(\kappa_{i}, \kappa_{i+1}\right)$ to $\kappa_{i}$. Let $\mathbb{P}=\prod_{i \in \text { Ord }} \mathbb{P}_{i}$ be the set support proper class product ordered componentwise, and let $G$ be $V$-generic over $\mathbb{P}$.

$V[G]$, being a model of $\mathrm{AC}$, is not our desired choiceless symmetric inner model $N$ witnessing the conclusions of Theorem 1 . In order to define $N$, we first note that by the Product Lemma, for $i \in \operatorname{Ord}, G_{i}$, the projection of $G$ onto $\mathbb{P}_{i}$, is $V$-generic over $\mathbb{P}_{i}$. In addition, for each $\delta \in\left(\kappa_{i}, \kappa_{i+1}\right)$, let $\mathbb{P}_{i} \uparrow \delta=\left\{p \in \mathbb{P}_{i} \mid \operatorname{dom}\left(p_{i}\right) \subseteq \kappa_{i} \times \delta\right\}$, and let $G_{i} \uparrow \delta=\left\{p \in G_{i} \mid p \in \mathbb{P}_{i} \uparrow \delta\right\}$. Standard arguments show that $G_{i}\left\lceil\delta\right.$ is $V$-generic over $\mathbb{P}_{i} \uparrow \delta$. Next, for each ordinal $\alpha$, let $\mathcal{F}_{\alpha}=\prod_{i<\alpha}\left(\kappa_{i}, \kappa_{i+1}\right)$ be the full support product of the open intervals $\left(\kappa_{i}, \kappa_{i+1}\right)$. Let $\mathcal{F}=\bigcup_{\alpha \in \text { Ord }} \mathcal{F}_{\alpha}$. For each $f \in \mathcal{F}$, $f=\left\langle\alpha_{i} \mid i<\alpha\right\rangle$, define $G \uparrow f=\prod_{i<\alpha}\left(G_{i} \uparrow \alpha_{i}\right)$. Observe that $G \uparrow f$ is $V$-generic over $\mathbb{P} \uparrow f=$

\footnotetext{
${ }^{3}$ The proof of [3, Theorem 1] proceeds by first forcing every member of the class of supercompact cardinals to be Laver indestructible. However, since the iteration used contains a low gap in Hamkins' sense of [8] and [9], by Hamkins' Gap Forcing Theorem of [8] and [9], it creates no new supercompact cardinals. This means that we may assume without loss of generality that all ground model supercompact cardinals are indestructible, and that there is no inaccessible limit of supercompact cardinals in the ground model.
} 
$\prod_{i<\alpha}\left(\mathbb{P}_{i}\left\lceil\alpha_{i}\right)\right.$. In other words, every $f$ is a set sequence of ordinals each of whose elements is a member of a unique open interval of the form $\left(\kappa_{i}, \kappa_{i+1}\right)$, and every $G_{i}\left\lceil\alpha_{i}\right.$ collapses each cardinal in the open interval $\left(\kappa_{i}, \alpha_{i}\right)$ to $\kappa_{i}$. $N$ can now be intuitively described as the least model of ZF extending $V$ which contains, for each $f \in \mathcal{F}$, the set $G \uparrow f$.

In order to define $N$ more formally, we let $\mathcal{L}_{1}$ be the ramified sublanguage of the forcing language $\mathcal{L}$ with respect to $\mathbb{P}$ which contains symbols $\check{v}$ for each $v \in V$, a unary predicate symbol $\check{V}$ (to be interpreted $\check{V}(\check{v}) \leftrightarrow v \in V$, i.e., $\check{V}$ allows us to determine members of the ground model), and symbols $\dot{G} \uparrow f$ for each $f \in \mathcal{F}$. $N$ is then defined as follows.

$$
\begin{aligned}
& N_{0}=\emptyset \text {. } \\
& N_{\delta}=\bigcup_{\alpha<\delta} N_{\alpha} \text { if } \delta \text { is a limit ordinal. } \\
& N_{\alpha+1}=\left\{\begin{array}{l|l}
x \subseteq N_{\alpha} & \begin{array}{l}
x \text { is definable over the model }\left\langle N_{\alpha}, \in, c\right\rangle_{c \in N_{\alpha}} \\
\text { via a term } \tau \in \mathcal{L}_{1} \text { of rank } \leq \alpha
\end{array}
\end{array}\right\} . \\
& N=\bigcup_{\alpha \in \operatorname{Ord}^{V}} N_{\alpha} \text {. }
\end{aligned}
$$

By [3, Lemmas $1.1-1.5$ and Lemma 1.7], $N \vDash$ "ZF + DC + Every successor cardinal is regular + Every limit cardinal is singular". Further, by [3, Lemmas $1.1-1.5]$, if $N \vDash$ " $\lambda$ is a successor cardinal", then $N \vDash " \lambda=\kappa$ for some $\kappa \in \mathcal{K}$ ". Our proof of Theorem 1 is therefore completed by the following lemma.

Lemma $1.1 N \vDash$ "Every successor cardinal satisfies the tree property".

Proof: Suppose $N \vDash " \kappa$ is a successor cardinal and $\mathfrak{T}$ is a $\kappa$-tree". As we have just observed, $\kappa \in \mathcal{K}$. Let $i_{0}$ be the unique ordinal such that $\kappa=\kappa_{i_{0}}$. Since $\mathfrak{T}$ may be coded by a set of ordinals, by [3, Lemma 1.1], we can assume that $\mathfrak{T} \in V\left[G\lceil f]\right.$ for some $f \in \mathcal{F}, f=\left\langle\alpha_{i} \mid i<j\right\rangle$. Note that if we wish, by padding if necessary, we may assume without loss of generality that $j$ is arbitrarily large. Write $f=\left\langle\left\langle\alpha_{i} \mid i<i_{0}\right\rangle,\left\langle\alpha_{i} \mid i_{0} \leq i<j\right\rangle\right\rangle=\left\langle f_{1}, f_{2}\right\rangle$. Represent $G \uparrow f$ as $\left(G \nmid f_{1}\right) \times(G \uparrow$ $\left.f_{2}\right)=G_{1} \times G_{2}$, where $G_{1} \times G_{2}$ is $V$-generic over the partial ordering $\mathbb{P}_{1} \times \mathbb{P}_{2}=\left(\mathbb{P} \nmid f_{1}\right) \times\left(\mathbb{P} \nmid f_{2}\right)$. If $V \vDash$ " $\kappa$ is supercompact", then because each $V$-supercompact cardinal is indestructible and $G_{2}$ is $V$-generic over $\mathbb{P}_{2}$, a partial ordering which is $\kappa$-directed closed, $V\left[G_{2}\right] \vDash$ " $\kappa$ is supercompact". Since by our assumptions on $V, V \vDash$ " $\kappa$ is not a limit of supercompact cardinals", it follows that $V \vDash "\left|\mathbb{P}_{1}\right|<\kappa "$. Therefore, by the Lévy-Solovay results [12], $\kappa$ remains supercompact in 
$V\left[G_{2} \times G_{1}\right]=V\left[G_{1} \times G_{2}\right]$. Because $\kappa$ is supercompact in $V\left[G_{1} \times G_{2}\right], \kappa$ is weakly compact in this model as well and hence satisfies the tree property in $V\left[G_{1} \times G_{2}\right]$. This means that in $V\left[G_{1} \times G_{2}\right] \subseteq N$, there is a branch of length $\kappa$ through $\mathfrak{T}$. If, however, $V \vDash$ " $\kappa$ is a successor of a singular limit of supercompact cardinals" 4 , then because each $V$-supercompact cardinal is indestructible and $G_{2}$ is $V$-generic over a partial ordering which is $\kappa$-directed closed, $V\left[G_{2}\right] \vDash$ " $\kappa$ is a successor of a singular limit of supercompact cardinals". As we have just observed, if $V \vDash$ " $\lambda<\kappa$ is supercompact", then $V\left[G_{1} \times G_{2}\right] \vDash$ " $\lambda$ is supercompact". Thus, since each component partial ordering of $\mathbb{P}_{1}$ is appropriately directed closed, $\kappa$ is in $V\left[G_{2} \times G_{1}\right]=V\left[G_{1} \times G_{2}\right]$ a successor of a singular limit of supercompact cardinals. Hence, by Shelah's theorem of [13], $\kappa$ satisfies the tree property in $V\left[G_{1} \times G_{2}\right]$. This means that in $V\left[G_{1} \times G_{2}\right] \subseteq N$, there is once again a branch of length $\kappa$ through $\mathfrak{T}$. Therefore, in either situation, $N \vDash$ " $\kappa$ satisfies the tree property". This completes the proof of both Lemma 1.1 and Theorem 1.

We remark that our methods of proof will actually allow $\mathbb{P}$ to be taken as a countable support product. However, for consistency with [3], we use set support in the definition of $\mathbb{P}$. Further, it is possible to begin the construction of $N$ by symmetrically collapsing $\kappa_{0}$ to be $\aleph_{2}$ instead of $\aleph_{1}$. If this is done, then the arguments of [3] in conjunction with standard techniques show that $N \vDash$ "ZF $+\mathrm{DC}_{\omega_{1}}+2^{\aleph_{0}}=\aleph_{1}+$ The tree property fails at $\aleph_{1}+$ Every successor cardinal is regular + Every limit cardinal is singular + Every successor cardinal greater than $\aleph_{1}$ satisfies the tree property". In this way, $N$ is slightly more "choice like", although by its construction, the full Axiom of Choice of course still fails in $N$. In addition, an alternate way of inferring that if $V \vDash$ " $\kappa$ is supercompact", then $N \vDash " \kappa$ satisfies the tree property" is to note that by [3, Lemma 1.2], $N \vDash$ " $\kappa$ is weakly compact". As we have already observed, this immediately implies that $N \vDash$ " $\kappa$ satisfies the tree property".

We end with two questions. First, we ask if the gap in consistency strength between the upper and lower bounds for the theory "ZF $+\mathrm{DC}+$ Every successor cardinal is regular + Every limit

\footnotetext{
${ }^{4}$ Note that by our assumptions on $V$, every limit of supercompact cardinals is singular.
} 
cardinal is singular + Every successor cardinal satisfies the tree property" provided in this paper can be narrowed somewhat. Finally, as was mentioned earlier, Theorem 1 does not answer our Question posed above in a ZFC context. We conclude by asking if a positive answer to this question can be found, or even if it is possible to construct a model of ZFC (starting from any suitable large cardinal hypotheses) combining the results of [6], [13], and [17], i.e., a model of ZFC in which every regular $\aleph_{i}$ for $1<i \leq \omega+1$ satisfies the tree property.

\section{References}

[1] U. Abraham, "Aronszajn Trees on $\aleph_{2}$ and $\aleph_{3}$ ", Annals of Pure and Applied Logic 24, 1983, $213-230$.

[2] A. Apter, "Some New Upper Bounds in Consistency Strength for Certain Choiceless Large Cardinal Patterns", Archive for Mathematical Logic 31, 1992, 201-205.

[3] A. Apter, "Some Results on Consecutive Large Cardinals", Annals of Pure and Applied Logic 25, 1983, 1-17.

[4] A. Apter, "Some Results on Consecutive Large Cardinals II: Applications of Radin Forcing", Israel Journal of Mathematics 52, 1985, 273-292.

[5] D. Busche, R. Schindler, "The Strength of Choiceless Patterns of Singular and Weakly Compact Cardinals", Annals of Pure and Applied Logic 159, 2009, 198-248.

[6] J. Cummings, M. Foreman, "The Tree Property", Advances in Mathematics 133, 1998, 1-32.

[7] M. Foreman, M. Magidor, R. Schindler, "The Consistency Strength of Successive Cardinals with the Tree Property", Journal of Symbolic Logic 66, 2001, 1837-1847.

[8] J. D. Hamkins, "Gap Forcing", Israel Journal of Mathematics 125, 2001, 237-252.

[9] J. D. Hamkins, "Gap Forcing: Generalizing the Lévy-Solovay Theorem", Bulletin of Symbolic Logic 5, 1999, 264-272. 
[10] T. Jech, Set Theory: The Third Millennium Edition, Revised and Expanded, Springer-Verlag, Berlin and New York, 2003.

[11] R. Laver, "Making the Supercompactness of $\kappa$ Indestructible under $\kappa$-Directed Closed Forcing", Israel Journal of Mathematics 29, 1978, 385-388.

[12] A. Lévy, R. Solovay, "Measurable Cardinals and the Continuum Hypothesis", Israel Journal of Mathematics 5, 1967, 234-248.

[13] M. Magidor, S. Shelah, "The Tree Property at Successors of Singular Cardinals", Archive for Mathematical Logic 35, 1996, 385-404.

[14] W. Mitchell, "Aronszajn Trees and the Independence of the Transfer Property", Annals of Mathematical Logic 5, 1972/73, 21-46.

[15] R. Schindler, "Weak Covering and the Tree Property", Archive for Mathematical Logic 38, 1999, 515-520.

[16] J. Silver, "Some Applications of Model Theory in Set Theory", Annals of Mathematical Logic 3, 1971, 45-110.

[17] D. Sinapova, "The Tree Property at $\aleph_{\omega+1}$ ", submitted for publication to the Journal of Symbolic Logic. 